\title{
Transient activation of dopaminergic neurons during development modulates visual responsiveness, locomotion and brain activity in a dopamine ontogeny model of schizophrenia
}

\author{
B Calcagno ${ }^{1}$, D Eyles ${ }^{1,2}$, B van Alphen ${ }^{1}$ and B van Swinderen ${ }^{1}$
}

It has been observed that certain developmental environmental risk factors for schizophrenia when modeled in rodents alter the trajectory of dopaminergic development, leading to persistent behavioural changes in adults. This has recently been articulated as the "dopamine ontogeny hypothesis of schizophrenia". To test one aspect of this hypothesis, namely that transient dopaminergic effects during development modulate attention-like behavior and arousal in adults, we turned to a small-brain model, Drosophila melanogaster. By applying genetic tools allowing transient activation or silencing of dopaminergic neurons in the fly brain, we investigated whether a critical window exists during development when altered dopamine (DA) activity levels could lead to impairments in arousal states in adult animals. We found that increased activity in dopaminergic neurons in later stages of development significantly increased visual responsiveness and locomotion, especially in adult males. This misallocation of visual salience and hyperactivity mimicked the effect of acute methamphetamine feeding to adult flies, suggesting up-regulated DA signaling could result from developmental manipulations. Finally, brain recordings revealed significantly reduced gamma-band activity in adult animals exposed to the transient developmental insult. Together, these data support the idea that transient alterations in DA signaling during development can permanently alter behavior in adults, and that a reductionist model such as Drosophila can be used to investigate potential mechanisms underlying complex cognitive disorders such as schizophrenia.

Translational Psychiatry (2013) 3, e206; doi:10.1038/tp.2012.139; published online 8 January 2013

\section{Introduction}

One of the most enduring hypotheses in schizophrenia research is the proposal that dopamine (DA) signaling is in some way abnormal in patients. ${ }^{1}$ In addition, there is a wealth of epidemiological data, indicating that adverse events occurring during either gestation or at birth can increase disease risk in offspring. ${ }^{2}$ In order to understand the critical neurodevelopmental abnormalities behind this epidemiology, the research community has developed a variety of animal models, usually in rodents. ${ }^{3}$ As adults, the animals resulting from these models frequently display brain functional and behavioural abnormalities consistent with aberrant DA signaling. The central, and to date yet unanswered question remains, how could in utero exposure to specific (environmental) insults induce persistent dopaminergic abnormalities in domains such as locomotion, response to DA releasing or blocking drugs, sensorimotor-gating deficits or deficits in attention-like behaviours in the adult?

Recent studies using rodent models of maternal vitamin $D$ deficiency or maternal infection, both proven epidemiological risk factors for schizophrenia, have provided convergent evidence that the early expression of genes crucial for the normal growth and differentiation of DA neurons in the central nervous system (CNS) may be altered. ${ }^{4,5}$ This has led to the proposal that the origins of a range of defective adult behaviours may be due to early alterations in DA ontogeny. ${ }^{6}$ Although rodent models will continue to be useful in providing answers as to how genetic and environmental factors shape the developing brain and adult behaviour, other reductionist approaches commonly used in developmental neuroscience have added advantages of faster turnover, efficient genetics, and precise temporal and spatial control of gene expression in a simpler nervous system. ${ }^{7}$ These alternate approaches may thus allow researchers to investigate molecularly any lead provided by clinical or genetic investigations. An example of this is the work of Sawa and co-workers who have described behavioural defects in the fruit fly Drosophila melanogaster expressing DISC1, a gene associated with an increased risk of schizophrenia. ${ }^{8}$ For these reasons we have chosen to investigate how interfering with normal DA ontogeny at various developmental stages of fly brain development could affect adult behaviour and brain function.

DA is a key behavioural modulator in all animals, ${ }^{9}$ and this is no different in the fly. Studies in Drosophila have supported the various roles that biogenic amines have in modulating sleep/wake behaviour, ${ }^{10,11}$ responsiveness to stimuli, ${ }^{12}$

\footnotetext{
${ }^{1}$ Queensland Brain Institute, The University of Queensland, St. Lucia, QLD, Australia and ${ }^{2}$ Queensland Centre for Mental Health Research, The University of Queensland, Wacol, QLD, Australia

Correspondence: Dr Bruno van Swinderen, Queensland Brain Institute, The University of Queensland, Upland Road, St. Lucia, QLD, Australia.

E-mail: b.vanswinderen@uq.edu.au

Keywords: brain development; dopamine; Drosophila; gamma; schizophrenia

Received 9 November 2012; accepted 10 November 2012
} 
learning and memory, ${ }^{13}$ and even attention-like behaviour. $^{10,14}$ Indeed, a recent comprehensive review of DA manipulations in Drosophila concluded that behavioural responsiveness to any stimuli (for example, olfactory, mechanical, visual) is highly dependent on optimal DA function, where any deviation from that DA optimum leads to altered responsiveness levels and most likely maladaptive behaviour. ${ }^{15}$ This observation in an insect model is reminiscent of the situation regarding the putative role of DA in the optimal tuning of prefrontal cortex (PFC) activity and attention in primates. ${ }^{16}$ In the following study, we first demonstrate that ethologically normal variability in DA content in wild-type flies correlates with performance in a visual paradigm. We then exploit the accessibility of Drosophila gene expression systems to examine whether transiently altering DA function during development permanently changes behaviour in adults. To date, there has been no investigation of how transient manipulations of DA function during various stages of Drosophila development could affect adult brain function and behavior, although one study suggested that long-term blockade of DA might compromise visual attention in flies, ${ }^{17}$ and a more recent study testing the schizophrenia susceptibility gene $d y$ sbindin described an effect on glutamate and DA function in larval and adult animals. ${ }^{18}$ Here, we selectively target dopaminergic neurons in the fly using the Gal4/UAS gene expression system. ${ }^{19}$ After manipulating DA neuronal function during various developmental windows, we investigated how visual responsiveness, locomotion and arousal are affected in adults. We find that there is a critical window of fly development, where transiently increasing DA activity alters visual behavior and general arousal in adult animals. Interestingly, these behavioural defects are associated with reduced gamma-like $(30-90 \mathrm{~Hz})$ activity in the adult fly brain. Given that deficits in locomotion and attention reflect both the positive and negative symptom profiles observed in patients with schizophrenia, and that gamma-band synchrony is reduced in brain recordings from patients with schizophrenia, ${ }^{20}$ our findings not only support the DA ontogeny hypotheses of schizophrenia but also suggest that this hypothesis might be efficiently addressed in model systems such as Drosophila.

\section{Materials and methods}

Drosophila melanogaster strains. Fly stocks were reared at $22{ }^{\circ} \mathrm{C}, 50-60 \%$ humidity, $12 \mathrm{~h}: 12 \mathrm{~h}$ light:dark cycle on yeast-based standard media. Wild-type flies were Canton-S (CS) and select Gal4 and UAS strains were local stocks originally obtained from the Bloomington Drosophila Stock Center or other sources as referenced. We used the Gal4/ UAS gene expression system ${ }^{19}$ to control gene expression in dopaminergic neurons. A tyrosine hydroxylase (Th)-linked Gal4 insertion on the third chromosome (Th-Gal4), on a CS genetic background, was used to specifically target dopaminergic cells. ${ }^{21} T h$ is the rate-limiting enzymatic step in the biosynthesis pathway of DA, and in the Drosophila central nervous system, the same neurons have been found to be Th- and DA-immunoreactive. ${ }^{22}$ UAS-shibire encodes a thermolabile variant of dynamin ${ }^{23}$ that prevents endocytosis at elevated temperatures $\left(31^{\circ} \mathrm{C}\right)$, thereby silencing $\mathrm{DA}$ neurons in Th-Gal4/ UAS-shibire flies at this restrictive temperature, but allowing normal synaptic processes at the permissive temperature $\left(22^{\circ} \mathrm{C}\right)$. UAS-TrpA1 encodes a temperature-sensitive cation channel that increases neuronal excitability at elevated temperatures $\left(31^{\circ} \mathrm{C}\right),{ }^{24}$ thereby activating DA neurons in Th-Gal4NAS-TrpA1 flies, but returning to basal activity levels at $22^{\circ} \mathrm{C}$. To visualize whether there was any gross alteration in DA circuits as a result of activation of these neurons during development, we used homozygous UAS-mCD8-green fluorescent protein (GFP); Th-Gal4 flies, which express a GFP marker in Thpositive neurons and crossed these to UAS-TrpA1 or control animals to generate progeny that were treated in the same way as described above.

Developmental interventions. Groups of 30-40 homozygous Th-Gal4 flies were crossed to 30-40 UAS-shibire or UAS-TrpA1 flies (crosses were balanced to cancel possible maternal effects), and allowed to lay eggs for 2 days on standard fly media in bottles, for 3-4 successive broods in different bottles. Progeny from these crosses and relevant genetic controls (either strain crossed to CS), were transferred to $31{ }^{\circ} \mathrm{C}$ for 2.5 days for one of four developmental epochs under investigation, lasting 2.5 days each: 0-2.5 days, $2.5-5$ days, $5-7.5$ days and $7.5-10$ days. For the rest of development and post-eclosion (adulthood) these strains remained at $22^{\circ} \mathrm{C}$, which is the normal housing temperature. To test for any developmental effects post-eclosion, flies were collected 0-6 h after emerging from the pupal stage, put in fresh media vials and transferred to $31^{\circ} \mathrm{C}$ either from days 0-2.5 post-eclosion (10-12.5 post embryo) or days $2.5-5$ post-eclosion (12.5-15 post embryo), then returned to $22^{\circ} \mathrm{C}$ until behavioural testing.

Visual behavior. Flies were collected under $\mathrm{CO}_{2}$ anaesthesia the day before visual experiments, separated by sex and transferred to loading tube (Jumbo pipettes, Thermo Fisher Scientific, Scoresby, Victoria, Australia) supplied with a drop of distilled water ( $\sim 30$ flies per tube). Tubes were kept in a circadian-controlled $22^{\circ} \mathrm{C}$ incubator overnight, and experiments were performed the following morning. Visual responsiveness was tested behaviorally using an automated visual choice maze design, described previously. ${ }^{25}$ Briefly, the device consists of 3-mm-wide paths grooved into a $28 \times$ $19 \times 1 \mathrm{~cm}$ acrylic slab placed groove-face down on a glass plate to form a closed horizontal choice platform. The platform was positioned $3 \mathrm{~cm}$ above an up-ended 19-inch flat-screen computer monitor (Mitsubishi Diamondpro 930, Itasca, IL, USA), on which was displayed a green/black grating of $1-\mathrm{cm}$ spatial resolution, moving at $3 \mathrm{~Hz}$. Flies introduced into the maze ( $\sim 30$ at a time) progress through eight left-right choices, to terminate into one of nine end tubes, where they are vacuumed and automatically counted by infra-red beam-crossing devices (Trikinetics, Waltham, MA, USA). Custom-made Matlab software (The MathWorks, Natick, MA, USA) tallies the fly distributions across the nine end tubes, to determine the visual response (VR) to the moving grating, by a weighted average. ${ }^{26}$ Positive VR indicate that flies turned in the same direction as the moving grating displayed beneath them. All maze runs were 
a
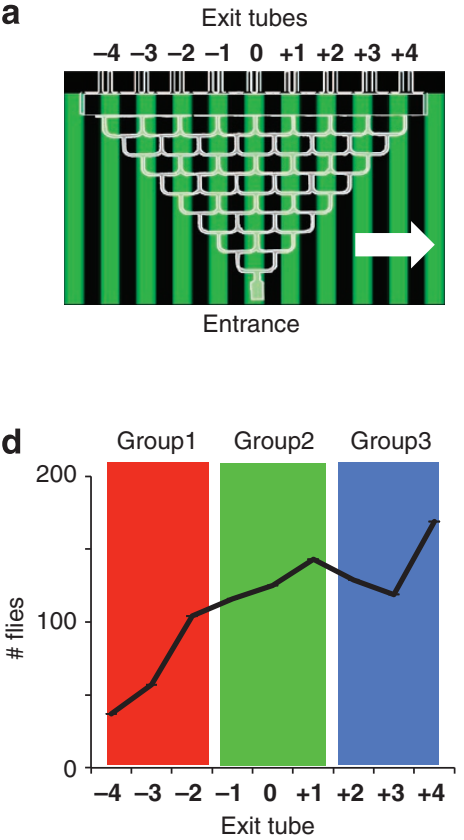

b

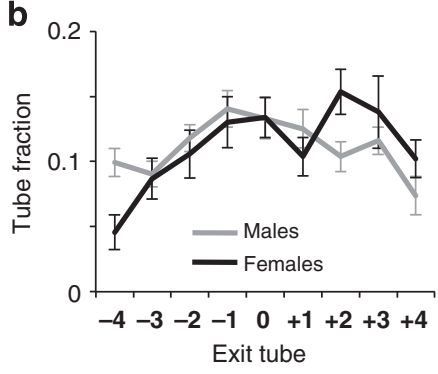

e

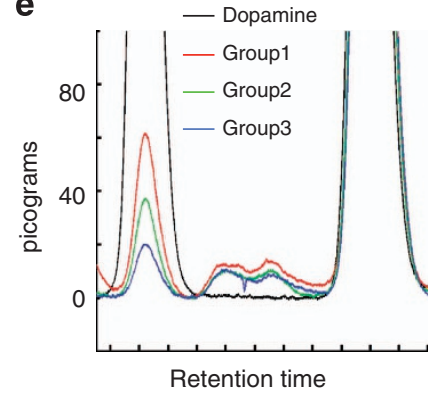

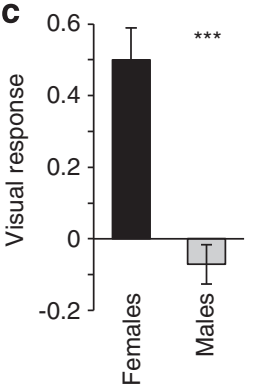

f

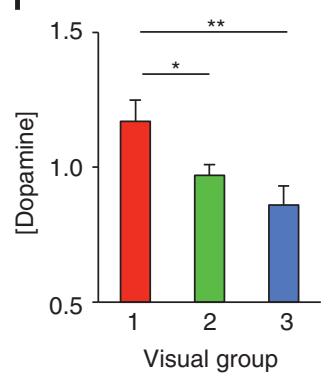

Figure 1 Visual responsiveness. (a) Flies walk through a choice maze while being exposed to moving gratings displayed on a computer monitor beneath them. The direction of grating motion (white arrow) influences their turning decisions at each choice point, and the population distribution among nine exit tubes determines the visual responsiveness of a strain. Positive responses indicate that flies are turning in the same direction as the grating. (b) Fly distributions among the nine exit tubes are plotted (proportion \pm s.e.m) for male and female wild-type flies ( $\mathrm{N}=200$ flies for each, comprising 8 maze runs of 25 flies). (c) VR ( \pm s.e.m.) is calculated as a weighted average of exit tube fractions; ${ }^{* * *}$ significantly different responses between males and females, $P<0.001$, by $t$-test comparing means ( $N=8$ runs of 25 flies per sex). (d) 999 wild-type female flies were run through the maze on three different days and collected into one of three visual responsiveness groups, depending on which exit tube they emerged from. Flies were immediately frozen for HPLC as they emerged from the maze. (e) Sample HPLC trace for flies from the three behavioural groups, as indicated by colour. Control DA peak is indicated (black). The large peak on the right is the internal standard for each sample. (f) Averaged normalized HPLC data for the three maze groups from (d). Each group includes data from nine different pools of five heads collected on three different days (see Materials and methods). ${ }^{* *} P<0.01$; ${ }^{*} P<0.05$, by $t$-test comparing means.

balanced for grating direction (forward and reverse), and weighted averages from different mazes (four were run simultaneously) were combined to yield a VR score for a strain. In a subset of experiments, we collected flies for high performance liquid chromatography (HPLC) analyses (see below) following visual experiments. For a total of 999 flies run over three separate experiments (Figure 1d), we selected 417 flies that ended in end tubes $+2,+3$ or +4 (thereby making mostly positive optomotor choices; Figure 1d blue area), 384 flies that ended in tubes $-1,0$ or 1 (thereby making mostly random choices; green area) and 198 flies that ended in tubes $-4,-3$ or -2 (thereby making mostly negative optomotor choices; red area). A random sample of five flies was taken from each of the three tubes per behavioural group (1-3), over three separate experiments. Thus, $(3 \times 15)$ or 45 flies per group were decapitated, and head DA content assessed by HPLC, Figure $1 e$.

Arousal and locomotion behavior. Fly locomotion was measured by two different techniques, either by counting infra-red beam crossing using Drosophila Activity Monitor Systems (Trikinetics) or by video tracking. For either technique, flies were placed individually into small $65-\mathrm{mm}$ glass tubes (Trikinetics), supplied on one end with standard food media. To measure long-term arousal behavior, epochs without any movement $>5$ min were tallied. This duration of inactivity has been associated with sleep in Drosophila, ${ }^{27}$ and by analyzing activity data this way, sleep metrics such as average time immobile or number of rest bouts per hour can be tallied. ${ }^{28}$ To measure activity in active, wake flies, we relied on video tracking of flies in the same tubes. Seventeen flies were filmed at a time using a webcam (Logitech, Strathfield South, NSW, Australia), and locomotion data were processed with custom-written Matlab software. Flies were filmed for $1-2 \mathrm{~h}$ during the day to determine average locomotion behavior, calculated as the number of pixels that a fly moved within the glass tube, per minute. Comparative recordings were also made during the flies' subjective night, to determine whether circadian effects on locomotion were altered in mutants.

Methamphetamine and haloperidol exposure. Groups of 30 flies at a time were provided overnight with a $20-\mu$ drop of $1.25 \mathrm{mg} \mathrm{ml}^{-1}$ methamphetamine-HCL (Sigma, St. Louis, $\mathrm{MO}$, USA) or $1 \mathrm{mM}$ haloperidol (Sigma) diluted in $1 \%$ sucrose solution (Andretic et al. ${ }^{10}$ for methamphetamine dosage experiments in flies, and Chang et al. ${ }^{29}$ for haloperidol dosage in flies), within their plastic loading tube for visual maze experiments. Ingestion of the drug was verified by adding blue food colouring to the drug solution; close to $100 \%$ of flies consumed the drug by the following morning, evident by counting flies with blue abdomens. Sham controls were also provided with a $1 \%$ sucrose water droplet, but without any drug added.

High performance liquid chromatography. The analysis of DA concentrations in the fly head was measured using 
HPLC with electrochemical detection. This system consisted of an autosampler and an isocratic HPLC pump (Model 1100, Agilent Technologies, Santa Clara, CA, USA), a Sunfire C18 column, $4.6 \times 150 \mathrm{~mm}, 5 \mu \mathrm{m}$; (Waters Corporation, Milford, MA, USA) and a Coulochem III (ESA Laboratories, MA, USA) electrochemical detector. Electrochemical detection was obtained with the working electrode set to a potential of $+300 \mathrm{mV}$ relative to the $\mathrm{Ag} / \mathrm{AgCl}$ reference electrode. $\mathrm{A}$ citrate buffer mobile phase solution was prepared with deionized water and degassed by vacuum filtration along with all solvents through $0.22-\mu \mathrm{m}$ cellulose membranes. The mobile phase consisted $25 \mathrm{~mm}$ sodium dihydrogen phosphate anhydrous, $50 \mathrm{~mm}$ citric acid monohydrate, $1.4 \mathrm{~mm}$ octane sulphonic acid and $1 \mathrm{mM}$ EDTA, adjusted with sodium hydroxide to attain a $\mathrm{pH}$ level of 5.5 , followed by the addition of $6 \%$ acetonitrile. Neurotransmitter standards were prepared with deionized water and consisted of $0.1 \mathrm{M}$ perchloric acid and $50 \mathrm{ng} \mathrm{ml}^{-1}$ deoxyepinephrine (DE). The standard monoamine solution was made with the internal standard solution DE and contained $5 \mu \mathrm{g} \mathrm{ml}^{-1}$ of DA. Insects taken from behavioural experiments were immediately frozen at $-20^{\circ} \mathrm{C}$. Fly heads were then dissected in groups of five for HPLC analysis on the morning of the experiment. Heads were placed into $100 \mu \mathrm{l}$ of DE solution and were homogenized via sonication $(3 \times 8 \mathrm{sec})$, followed by centrifugation at $13000 \mathrm{rpm}$ for $5 \mathrm{~min}$ at $4{ }^{\circ} \mathrm{C}$. Samples were then syringe filtered and a fixed volume of $10 \mu$ of the resultant supernatant was injected directly into the chromatographic system. Data was quantified with ChemStation software (Agilent Technologies) by calculating peak-height ratios of each compound relative to the internal standard DE and corrected for dilution. Compound identity was determined by retention time and the final amount of neurotransmitter expressed as picograms per head. As DA levels varied between experimental batches of flies, for maze experiments done on separate days, DA concentrations were normalized to the entire maze average on the day of testing (five heads per each of nine end tubes).

Electrophysiology. Local field potentials (LFPs) were recorded from the Drosophila brain while the tethered flies were exposed to visual stimuli, as described previously. ${ }^{30,31}$ Flies were tethered to tungsten posts with light-cured dental cement (Coltene Whaledent, Altstätten, Switzerland), and a 25- $\mu \mathrm{m}$ tungsten wire implanted into the fly thorax served as electrical grounding. LFPs were recorded as a voltage differential between two glass electrodes implanted into either hemisphere of the fly brain, $\sim 100 \mu \mathrm{m}$ down from the dorsal rim of either eye. LFPs were sampled at $300 \mathrm{~Hz}$, preamplified with field-effect transistors (NBLabs, Denison, TX, USA) and further amplified with a DP201 differential amplifier (Warner Instruments, Hamden, CT, USA), and digitized with a custom-written Labview program, through a National Instruments data acquisition board. A visual stimulus (a $15^{\circ}$-wide unlit bar moving clockwise $120^{\circ}$ per second on a cylinder of $72 \times 24$ green light-emitting diodes) was recorded alongside the LFP as a voltage signal between 0 and 5.5 volts. The brain signal was analyzed in two different ways, as described previously. ${ }^{31}$ First, the average voltage amplitude was calculated and mapped across 24 sectors of the recurrent image rotation sequence; this is equivalent to the visual event-related potential (ERP) in human electroencephalograms (EEG). ${ }^{32}$ Second, the LFP signal was spectrally decomposed by Fourier analysis, and power for different frequency domains from $10-100 \mathrm{~Hz}$ was calculated using custom-written Matlab software.

Confocal imaging. Targeted expression of UAS-mCD8GFP in Th-Gal4/UAS-TrpA1-treated flies and genetic controls was achieved by crossing w;UAS-mCD8-GFP; Th-Gal4 to UAS-TrpA1 (or to wild-type flies, for the controls). After exposing flies to increased temperatures during the relevant epoch of developmental intervention, 4-day-old adult male brains (experimental and control) were free-hand dissected and placed on a cover-slip with Ringer solution $(108 \mathrm{~mm}$ $\mathrm{NaCl}, 5 \mathrm{~mm} \mathrm{KCl}, 8.2 \mathrm{~mm} \mathrm{MgCl} 2,4 \mathrm{~mm} \mathrm{NaHCO}, 1 \mathrm{~mm}$ $\mathrm{NaH}_{2} \mathrm{PO}_{4}, 2 \mathrm{~mm} \quad \mathrm{CaCl}_{2}$ and $5 \mathrm{~mm}$ HEPES $(\mathrm{pH} 7.5$, $265 \mathrm{mOsm}$ ) and immediately imaged. Confocal imaging of Drosophila brains was conducted using a Carl Ziess LSM510 confocal microscope (North Ryde, NSW, Australia). The 488$\mathrm{nm}$ laser line was used to excite GFP-activated dopaminergic neurons and observed with a $\times 10 / 0.3$ Plan-NEOFLUAR objective at a resolution of $2048 \times 2048$. Pixel resolution in the images was $0.77 \times 0.77 \mu \mathrm{m}$ with 37 slices imaged at $2.00-\mu \mathrm{m}$ intervals. DA clusters were identified based on previously published registration of distinct cell groups. ${ }^{33}$ Identification of cells within each cluster, or of a target structure, was determined for nine experimental brains and seven control brains.

Statistics. Behavioural data were analyzed for statistical significance using SPSS and prism software (GraphPad Software, La Jolla, CA, USA). A two-tailed $t$-test $(P<0.05)$ was used to assess differences between male and female wild-type CS flies. Linear, quadratic and cubic trend analysis with two-tailed $t$-test $(P<0.05)$ determined the significance of curve fits for the relationship between percentage of flies in each tube of the visual choice paradigm and individual DA concentrations. Correlation analysis (Pearson's $r$ ) examined strength of the relationship between percentage of flies and total DA levels for each individual tube of the optomotor maze paradigm, with two-tailed $t$-test $(P<0.05)$ indicating significant difference of the relationship. A series of one-way analysis of variance (ANOVA) between grouped means of 18 mazes of all transgenic mutants and controls over the four developmental epochs were conducted to examine behavioural data. Post-hoc Bonferroni's multiple comparison test was used to determine significant differences between transgenic mutants and controls within each of the four developmental windows. Post-hoc linear trend analysis $\left(r^{2}\right.$, $P<0.05)$ was also used to determine the relationship of transgenic mutants and pooled controls over all four developmental epochs.

\section{Results}

To measure visual responsiveness in Drosophila, we used a population paradigm where flies respond to moving gratings displayed on a computer monitor while running through a choice maze (Figure 1a, and Materials and methods). 
Surprisingly, we found that male and female flies displayed significantly different responsiveness to a $3-\mathrm{Hz}$ moving grating, with wild-type female flies responding positively (as shown previously, ${ }^{25}$ but wild-type males not responding, on average (Figures $1 \mathrm{~b}$ and $\mathrm{c}$ ).

Like humans, flies display considerable behavioural variability among individuals-even if these are drawn from the same "wild-type" stock. ${ }^{26}$ Considering the sensitivity of fly behaviors to DA levels, ${ }^{15}$ we suspected that differences in DA function might be a major causal factor. In a first set of experiments on wild-type, we therefore selected flies emerging from the maze following a visual experiment to measure, using HPLC (see Materials and methods), whether DA levels were differentially represented in wild-type flies making different choices in the maze (Figures $1 d-f$ ). We found that flies moving against the grating motion had significantly more DA in their heads than flies moving with the motion (Figure 1f, $P=0.009$ ), and also more DA than flies making neutral choices $(P=0.04)$. These results support other studies showing that DA levels in the fly brain modulate visual responsiveness. ${ }^{14,17}$ Although this result does not necessarily rule out a role for other neurotransmitters (for example, serotonin and octopamine) in modulating visual responsiveness in flies, it does highlight how variable DA levels can be among individual flies within a wild-type strain (GonzaloGomez et al. ${ }^{34}$ for similar recent observations tied to circadian rhythms). Coupled with the likelihood of genetic heterogeneity within a strain, ${ }^{26}$ developmental influences are another likely source of this variability; for example, environmental stressors have been shown to alter DA function in flies. ${ }^{35}$ To investigate a role for DA more directly, we therefore next tested whether increasing or decreasing DA activity during discrete developmental windows altered visual responsiveness in adult Drosophila.

Th is the rate-limiting enzyme in DA synthesis, in flies as well as other animals. ${ }^{36}$ To transiently manipulate DA function during development we exploited the Drosophila UAS/Gal4 gene expression system, ${ }^{19}$ using the Th-Gal4 line ${ }^{21}$ to drive expression of two different UAS effectors, UAS-TrpA $1^{24}$ and $U A S$-shibire ${ }^{23}$ that allowed transient activation or silencing of dopaminergic neurons, respectively (see Materials and methods). To determine whether there was a critical window of development during which either manipulations of DA activity modulated behaviour in the adult, we divided fly development into four quarters of 2.5 days each, with adult flies emerging around day 10; we then tested for visual responsiveness in the maze after 6 days of adulthood (Figures $2 a-d)$. As fly brain development continues in early adulthood, ${ }^{37}$ we also manipulated DA activity in adult flies (days 10-12.5 and days 12.5-15), for comparison (Figure $3 b$ ).

Transient silencing of DA neurons during development had no significant effects on visual responsiveness in adult females (Figure 2a) or adult males (Figure 2c). In contrast, activating DA neurons dramatically increased visual responsiveness in adult males compared with similarly treated genetic controls, but only if activation was during the third or fourth quarter (Figure 2d, days 5-10), which corresponds to the pupal stage of fly development. This transient manipulation not only produced a positive optomotor VR in males, but also increased responsiveness to even higher levels than a
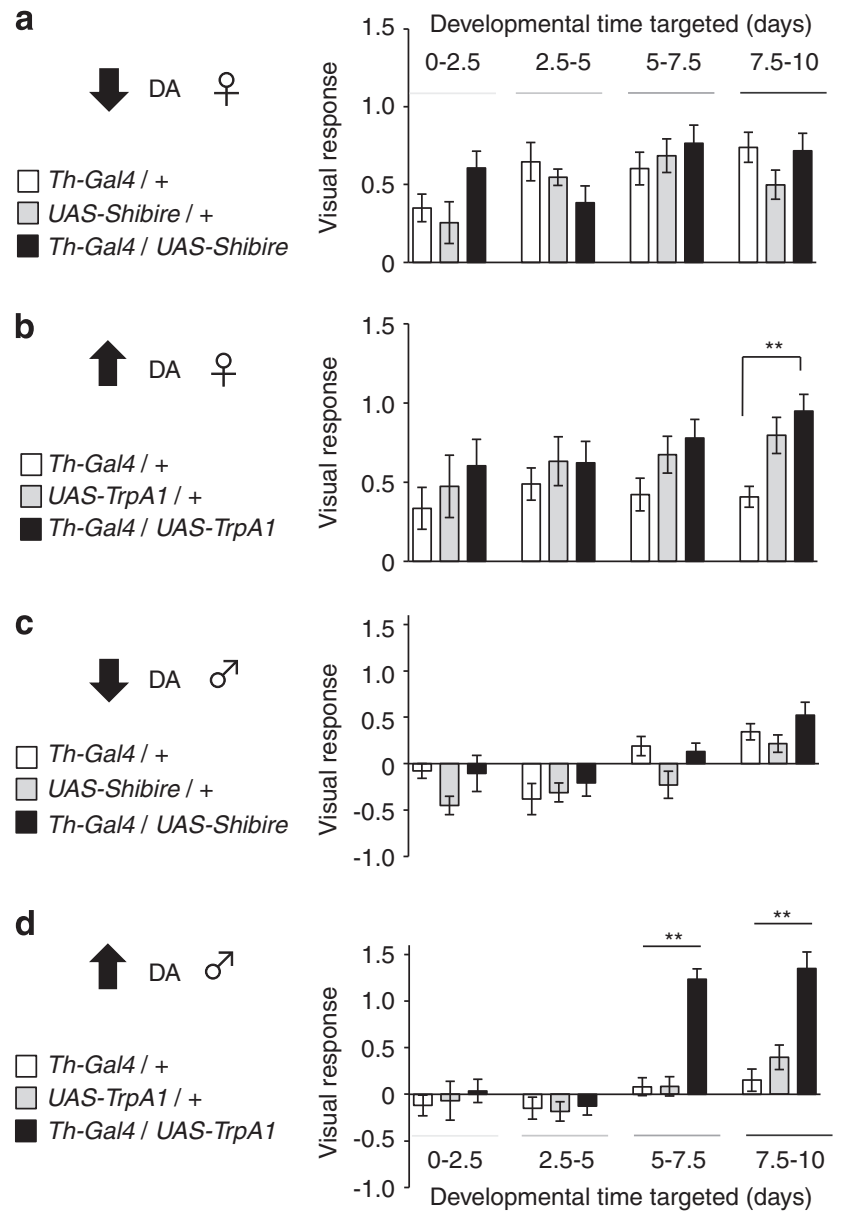

Figure 2 Effect of DA ontogeny on visual responsiveness in adults. (a, b) VR ( \pm s.e.m) in adult female flies in which DA function has been silenced (a) or activated (b) during various 2.5-day windows of development, as indicated by the graded grey bars. Black histograms: experimental group; white and grey histograms are genetic controls, as indicated, which have been subject to the same temperature shift (see Materials and methods). (c, d) VR ( \pm s.e.m) in adult male flies in which DA function has been silenced (c) or activated (d) during various 2.5-day windows of development. Black histograms: experimental group; white and grey histograms are genetic controls, as indicated, which have been subject to the same temperature shift (see Materials and methods). +, wild-type (CS) allele; Th-Gal4, Th enhancer trap; UAS-shibire, synaptic silencer trans-gene; UAS-TrpA1, activator of neural excitability ${ }^{*} P<0.01$, by ANOVA between grouped means of 18 maze experiments ( 450 flies) for all strains over the four developmental epochs, adjusted for multiple comparisons by a post-hoc Bonferroni test.

untreated females $(\mathrm{VR}=1.35 \pm 0.18$ for fourth quarter activated males versus $V R=0.5 \pm 0.09$ for untreated females). Female visual responsiveness was also increased by transient DA activation during the fourth quarter of development (Figure $2 b$ ), but this was not significant compared with one of the genetic controls (UAS-TrpA1/+). These results show that DA activation specifically during the pupal stage of fly development increases visual responsiveness in adults, and that this effect is most penetrant in males. In contrast to these transient developmental effects, chronic activation of DA throughout development using a different, constitutive neuronal activation tool (a truncated potassium channel, eag ${ }^{44138}$ in Th-Gal4/UAS-eag ${ }^{941}$ flies) did not 
increase visual responsiveness in males $(V R=-0.10 \pm 0.26$, $N=180$ flies).

Preceding behavioural experiments on wild-type flies revealed a significant association between visual responsiveness in the maze and DA levels in fly heads (Figure 1f): flies making positive choices were found to have less DA than flies making neutral or negative choices. As we were able to dramatically increase visual responsiveness via a developmental manipulation in transgenic strains, we next asked whether decreased DA levels and increased visual responsiveness were similarly correlated among our genetically manipulated strains. For this experiment, we focused on our fourth quarter DA-activated male and female groups, which produced the greatest increase in visual responsiveness compared with controls (Figures $2 b$ and $d$ ). We found that visual responsiveness was negatively correlated with DA concentration in these strains (Figure 3a, $r=-0.82$, $P<0.05)$. This result in our manipulated flies is consistent with our wild-type data, indicating that decreased DA levels are associated with increased visual responsiveness, whereas increased DA levels seem to abolish the positive response. Interestingly, our genetically manipulated males showed the greatest decrease in DA levels, and the greatest associated increase in visual responsiveness (Figure 3a, black square). This decrease in DA content in the heads of manipulated males was not associated with any gross changes in DA neuroanatomy, as visualized by GFP labelling of Th-expressing neurons in treated animals and controls (Figure 4).

In Drosophila, the brain continues to develop past the pupal stage and into the first 3 days of adulthood. Indeed, 1-3-dayold flies are physiologically immature, unable to learn as well as older adults and exist in a generally lower arousal state, ${ }^{27,37}$ further indicating that the fly brain is still extensively rewiring itself past eclosion from the pupal stage. To test whether the DA activation of the young adult brain also affected visual responsiveness, we transiently increased DA activity in Th-Gal4/UAS-TrpA1 flies and their controls for 2.5 days past eclosion (days 10-12.5). Consistent with the view that the developing male brain is sensitive to DA activation levels, this manipulation also significantly increased visual responsiveness (Figure $3 \mathrm{~b}$ ). In contrast, transiently increasing DA activity in older male Th-Gal4/UAS-TrpA1 flies (during days 2.5-5 post-eclosion, or days 12.5-15) had no effect on visual responsiveness (Figure $3 b$ ). These results delineate a surprisingly broad ( $\sim 7$ days) window of developmental vulnerability, where DA activation significantly alters visual responsiveness in adult male flies. However, and most importantly, our negative results in older males highlights the strict developmental nature of this intervention.

We next investigated the effect of methamphetamine and haloperidol on our manipulated animals. Methamphetamine is a psychomimetic drug that increases DA levels in brain synapses, ${ }^{39}$ resulting in hyperactivity, fragmented sleep and altered salience effects. In flies, methamphetamines have been shown to similarly increase general arousal. ${ }^{10}$ Haloperidol is an antipsychotic drug that targets D2-like receptors, ${ }^{40}$ in flies as well as mammals. ${ }^{29,41}$ We found that exposing adult (6-day-old) flies to methamphetamine (see Materials and methods) increased visual responsiveness in our individual
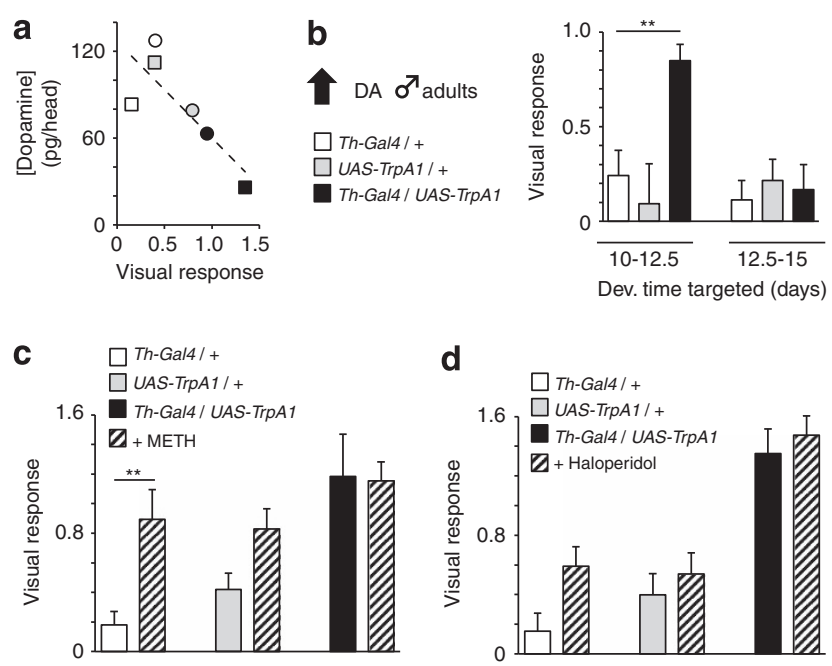

Figure 3 Other dopaminergic manipulations. (a) Head DA content and corresponding average visual responsiveness of fourth quarter-treated adult male (squares) and female (circles) Th-Gal4/UAS-TrpA1 flies (black) and genetic controls (white, Th-Gal4/ +; grey, UAS-TrpA1/ + ). Each HPLC data point represents one pooled sample of five heads, whereas the behavioural data represents the population average for that strain/condition, taken from Figure $2 \mathrm{~b}$ and $\mathrm{d}$. Dashed line, linear regression of data, $R=-0.82, P<0.05$. (b) VR ( \pm s.e.m) in adult male flies in which DA function has been activated during two 2.5-day windows of posteclosion adulthood (black histograms). Similarly treated genetic controls are indicated, in white and grey. ${ }^{\star \star} P<0.01$, by ANOVA between grouped means, adjusted for multiple comparisons by a post-hoc Bonferroni test. (c) Methamphetamine $\left(1.25 \mathrm{mg} \mathrm{ml}^{-1}\right)$ feeding effects on the VR $( \pm$ s.e.m) in fourth quarter DAmanipulated male flies and similarly treated genetic controls. ${ }^{*} P<0.01$, by $t$-test comparing means. $N=16$ experiments ( 400 flies) (d) Haloperidol ( $1 \mathrm{mM}$ ) feeding effects on the VR ( \pm s.e.m) in fourth quarter DA-manipulated male flies and similarly treated genetic controls. $N=16$ experiments ( $\sim 400$ flies).

Th-Gal4/ + and UAS-TrpA1/ + control strains (Figure 3c), which is consistent with a previous report showing similar effects in wild-type females. ${ }^{26}$ However, exposing our developmental DA-manipulated adults (Th-Gal4/UAS-TrpA1) to methamphetamine did not increase visual responsiveness any more than the already high level seen in these flies (Figure $3 \mathrm{c}$ ). This suggests that the transient developmental manipulation in late pupal development produces a ceiling effect with regards to DA signaling and its effect on visual responsiveness in adult males, potentially mimicking the effect of methamphetamine at DA synapses. ${ }^{39}$ Feeding the antipsychotic drug haloperidol did not significantly alter visual responsiveness in our developmental DA-manipulated adults or controls (Figure $3 d$ ), suggesting that, unlike in mammalian models of the schizophrenia centred on the D2 receptor function, ${ }^{42}$ a different DA receptor may be involved in our Drosophila model of the disorder.

We questioned whether visual responsiveness was the only behaviour altered by developmental activation of DA. Biogenic amines such as DA are crucial for regulating activity levels and arousal states in flies as well as other animals, and DA in particular has been shown to be a wake-promoting neuromodulator (reviewed in van Swinderen and Andretic ${ }^{15}$ ). We therefore tested activity levels of Th-Gal4/UAS-TrpA1 males that were activated only during the fourth quarter of development. Similar to the effects of acute 

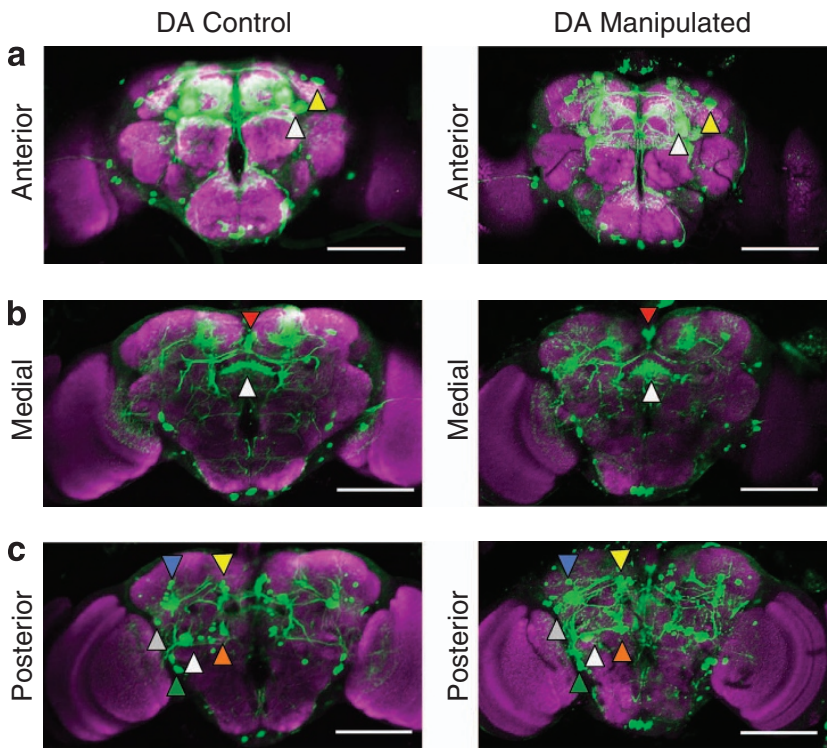

d

DA Control

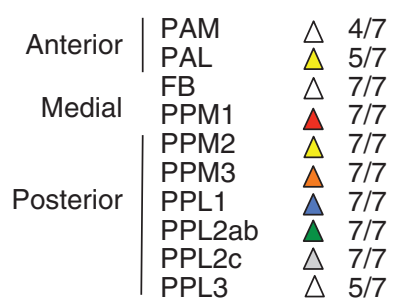

DA Manipulated
$\triangle 7 / 9$
$\triangle 9 / 9$
$\triangle 9 / 9$
$\triangle 9 / 9$
$\triangle 9 / 9$
$\triangle 8 / 9$
$\triangle 9 / 9$
$\triangle 8 / 9$
$\triangle 8 / 9$
$\triangle 8 / 9$

Figure 4 Imaging Th-expressing neurons in DA-manipulated flies and controls. Confocal imaging of UAS-GFP/UAS-TrpA1;Th-Gal4/+ males in which DA has been activated during the fourth quarter of development (DA manipulated), and similarly treated UAS-GFP/+;Th-Gal4/+ controls (DA control). Three vertical sections are shown (a, anterior, $\mathbf{b}$, medial and $\mathbf{c}$, posterior) for two sample brains (see Materials and methods). Magenta is nc82 antibody staining for synapses, green is Th-expressing neurons. Scale bar $=100 \mu \mathrm{m}$. (d) Summary data for seven DA control and nine DA-manipulated brains, indicating the proportion of brains where each of $10 \mathrm{DA}$ clusters or target structures were seen, as indicated on sample brains by coloured triangles. The DA clusters are as described in Mao and Davis ${ }^{33}$ minus three smaller clusters that were difficult to discern in either group (PPM4, PPD and PPL5). ${ }^{33} \mathrm{FB}$, fan-shaped body.

methamphetamine exposure, ${ }^{10}$ these animals displayed heightened locomotion, covering twice more distance per minute compared with controls (Figure 5a; see Materials and methods). Circadian analyses of activity data revealed that DA-manipulated animals also displayed fragmented sleep at night, with increase number of sleep bouts of shorter duration (Figures $5 b$ and $c$ ). Together, our arousal data therefore confirm that not only visual responsiveness is increased as a consequence of our developmental manipulation; animals are also hyperactive.

In order to further explore why developmental activation of DA in males might lead to increased visual responsiveness, we recorded the brain activity of flies exposed to moving visual stimuli (Figure 6a). Two glass electrodes were implanted into the brain of tethered male flies, similar to a previously reported preparation to record LFPs from behaving Drosophila ${ }^{31}$ (see Materials and methods). Flies were suspended inside a cylindrical arena upon which moving stimuli could be displayed with light-emitting diodes (for example, a $15^{\circ}$ black bar rotating around the fly at $120^{\circ}$ per second, on a lit green background, as in Figure 6a). Simultaneous recordings of LFPs and the virtual bar position (Figure 6b) allowed brain responsiveness to the visual object (measured as a voltage differential) to be signal-averaged as an ERP (Figure 6c). Surprisingly, selected male Th-Gal4/UAS-TrpA1 flies that displayed increased visual responsiveness to the moving grating in the choice maze paradigm (Figure 1) displayed significantly decreased LFP responsiveness to the moving bar, compared with similarly treated genetic controls (Figure 6d). We next examined whether certain frequency domains were altered in the LFP of our treated animals. Previous studies have shown that visual responses in Drosophila are associated with $20-30-\mathrm{Hz}$ LFP activity, and that oscillations in this frequency range increase when animals attend to visual stimuli. ${ }^{30,31}$ Treated Th-Gal4/UASTrpA1 male flies displayed decreased LFP power when presented with the moving visual stimulus (Figures $6 e$ and $f$ ), in both the lower $(30-50 \mathrm{~Hz})$ and higher $(50-90 \mathrm{~Hz})$ gamma range (Figure 6f). In addition, the characteristic $20-30-\mathrm{Hz}$ peak in the power spectrum of flies responding to a salient visual stimulus ${ }^{31}$ was abolished in DA-manipulated Th-Gal4/ UAS-TrpA1 flies (Figure 6e).

\section{Discussion}

Two of the most compelling hypotheses in schizophrenia research, namely the DA hypothesis and the neurodevelopmental hypothesis, can be synthesized into the one idea: that there are early events in DA development that predispose an individual to developing schizophrenia later in life. This has been previously articulated as the "dopamine ontogeny hypothesis" of schizophrenia. ${ }^{6}$ Previous support for this hypothesis comes from studies in different epidemiologically based neurodevelopmental rodent models. ${ }^{4,5}$ Here, we show support for this hypothesis using a simpler model animal, Drosophila melanogaster, and identify an epoch of brain development in which increasing DA activity leads to impairments in relevant behaviours in adult animals.

Simple animal models such as Drosophila can never fully address the symptoms of complex human psychiatric disorders such as schizophrenia. Diagnoses for such disorders require verbal reports and detailed psychiatric evaluations, which are of course impossible in any animal. However, as the underlying neurobiology for psychiatric disorders is complex, one way of understanding underlying mechanisms at the cellular and molecular level is via animal models. Traditionally, this has involved modeling genetic and environmental disease risk factors in rodents. Model systems commonly used in developmental neuroscience, such as mice, offer several advantages for understanding mechanisms underlying psychiatric disorders. ${ }^{43}$ However, relevant phenotypes can also be readily studied other model organisms that although possessing smaller brains are endowed with powerful genetic tools, such as flies, zebrafish, and even nematodes. $^{7}$

The positive symptoms of schizophrenia (for example, hallucinations) have been conceptualized as a defect in the allocation of salience. ${ }^{44}$ We found that increasing DA activity 

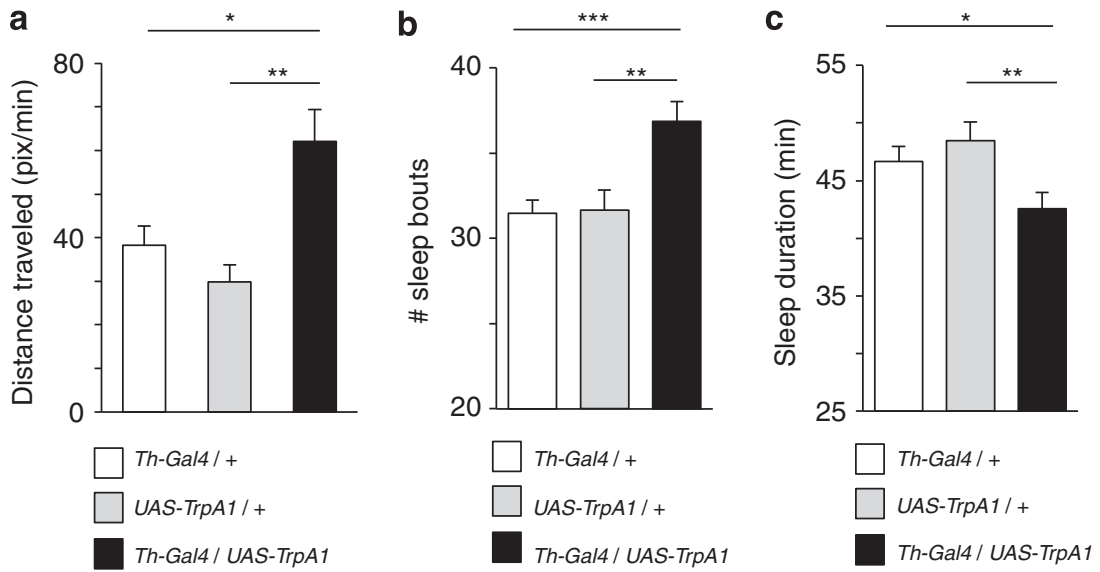

Figure 5 Activity levels and sleep. (a) Measure of locomotion activity (pixels per minute, see Materials and methods) in male flies that had increased DA activity during the fourth quarter of development (black histograms), compared with treated genetic controls (white and grey, as indicated). ${ }^{\star \star} P<0.01$, by $t$-test comparison of means. (b) The average number of periods of inactivity $>5 \mathrm{~min}$ ( \pm s.e.m) over a 24-h period, for male flies that had increased DA activity during the fourth quarter of development (black histograms), compared with treated genetic controls (white and grey, as indicated). (c) The average duration of quiescent periods (min \pm s.e.m) over $24 \mathrm{~h}$ for the same flies. ${ }^{* \star *} P<0.001 ;{ }^{* \star} P<0.01 ;{ }^{*} P<0.05$, by $t$-test comparison of means. $N=32$ flies per genotype.

during later stages of Drosophila development dramatically increases visual responsiveness in adult males, whereas decreased DA activity during the same critical window achieves no such effect. Such increased visual responsiveness in these strains potentially also represents a missallocation of salience. Another manipulation that increases visual responsiveness and arousal in Drosophila is acute methamphetamine feeding, ${ }^{10,26}$ further supporting a role for DA in regulating these behaviours. Interestingly, a third manipulation that similarly increased visual responsiveness levels in the same paradigm was seen in dunce ${ }^{1}$ mutants, ${ }^{30}$ which disrupt a gene that has been linked to schizophrenia in humans, cyclic AMP phosphodiesterase 4B (PDE4B). ${ }^{45}$ As dopaminergic neurons target PDE-expressing neurons in the Drosophila brain to control learning and memory processes, ${ }^{13}$ we may already be highlighting a simple circuit through which the effects of DA ontogeny on this behavior can be potentially understood.

Increasing DA activity during discrete periods of brain development can therefore phenocopy the effects of feeding adult flies a psychomimetic drug, methamphetamine, or of disrupting a schizophrenia candidate gene, PDE4B. Such phenocopying was seen not only for visual responsiveness, but also for other behavioural measures more traditionally associated with animal models of schizophrenia, such as hyperactivity. ${ }^{3}$ In addition, our developmental manipulation resulted in more fragmented sleep in adults, a phenotype previously seen in a Drosophila model of DISC1, a major schizophrenia candidate gene. ${ }^{8}$ This suggests that disrupted sleep architecture, in addition to hyperactivity, may be a common symptom in models of this disease. Crucially, DA activity has been intimately tied to arousal in Drosophila (reviewed in van Swinderen and Andretic ${ }^{15}$ ). As the morphology of Th-expressing neurons did not seem to be grossly impaired in our DA-manipulated flies (Figure 4), this might suggest that our controlled developmental insults alter more specific aspects of DA signalling, such as up-regulating postsynaptic receptor levels or pre-synaptic DA synthesis or release mechanisms, thereby increasing the sensitivity of DAmediated neuromodulation. Future experiments in Drosophila should resolve exactly which aspect of DA signaling has been altered, precisely which downstream neurons are involved, and how these might modulate arousal and visual salience in the fly brain.

To better elucidate whether attention-like processes could indeed be defective in our DA-manipulated males, we recorded their LFP brain activity as they were presented with a salient visual stimulus, a moving bar. Instead of finding increased LFP responsiveness to the bar, we saw the opposite: a decreased LFP response compared with similarly treated controls. This signal-averaged response is analogous to an ERP in human EEG studies. ${ }^{32}$ The fact that the VR in brain activity is smaller in the same flies that had displayed an increased behavioural responsiveness (animals used for electrophysiology were selected directly from the behavioural assay) suggests that improved vision cannot be proposed as a simpler explanation for their improved performance in the maze. Rather, another explanation is that attention-like processes are defective. For the similarly behaving PDE4B mutant, dunce, ${ }^{1}$ it has been previously proposed that increased visual responsiveness in the maze paradigm reflects a failure to suppress an optomotor response. ${ }^{30,46}$ Optomotor responses in flies, like optokinetic responses in humans, are visual-motor reflexes that promote stabilization of the visual field, especially in a dynamic environment. ${ }^{47}$ Attentional processes are required to suppress such reflexes, such as when a person must for example fixate on a visual target while driving. Such suppression mechanisms falter in older individuals, ${ }^{48}$ but also in some schizophrenia patients. ${ }^{49}$ Increased optomotor responsiveness in Drosophila may thus represent a similar failure of suppression mechanisms relevant to visual attention.

The most intriguing outcome of our manipulation of DA ontogeny in flies is that it is also associated with decreased oscillatory activity in the brain, in the lower and higher gamma range $(30-50 \mathrm{~Hz}$ and $50-90 \mathrm{~Hz}$, respectively). This is highly 
a

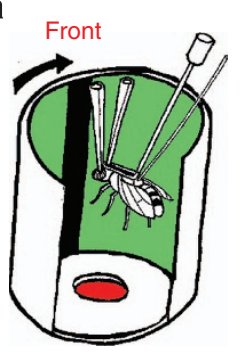

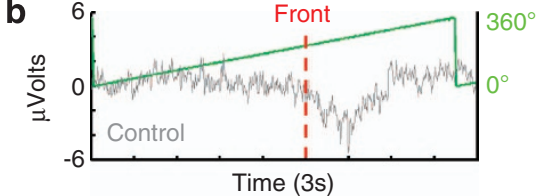

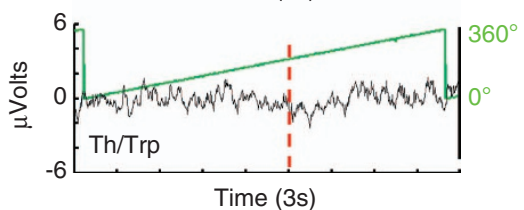

C
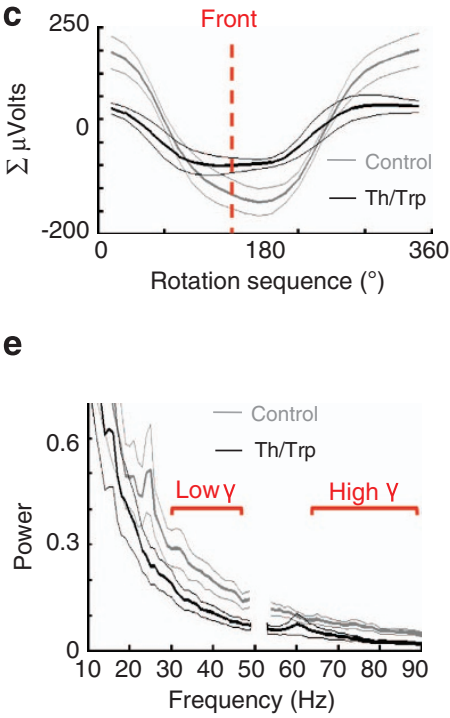
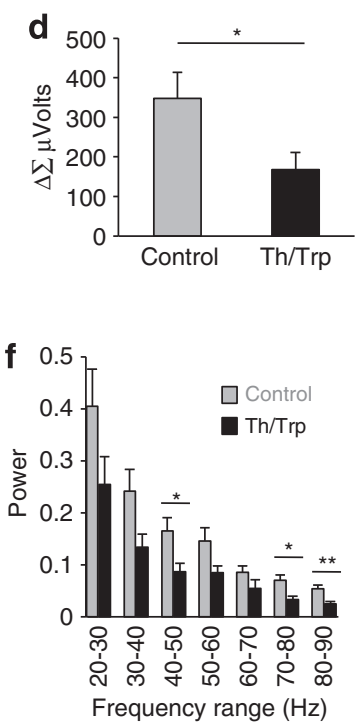

Figure 6 Electrophysiology. (a) A tethered fly is positioned in the centre of a light-emitting diodes arena. A dark bar displayed on the arena wall moves around the fly with a period of $3 \mathrm{~s}$. LFPs are recorded as a voltage differential between two electrodes implanted into the fly brain. (b) LFP traces shown together with image position (green line represents the image rotation sequence between 0 and $360^{\circ}$ ). Three seconds of activity are shown, representing LFP activity for one full rotation $\left(360^{\circ}\right)$ of the image. Dashed red line: time when the moving bar is in front of the fly. Upper panel, sample LFP activity from a UAS-TrpA1/ + control male fly; bottom panel, sample activity from a Th-Gal4/UAS-TrpA1 male fly in which DA function has been activated during the fourth quarter of development. (c) Average voltage ( \pm s.e.m.) from the LFP (see Materials and methods) for fourth quarter treated ThGal4/UAS-TrpA1 male flies (grey line) and similarly treated controls (data from ThGal4/ + and UAS-TrpA1/ + are combined). (d) Average amplitude of voltage deflections $\left(\Delta \Sigma \mu\right.$ volts \pm s.e.m.) from for the same data as in (c). ${ }^{*} P<0.05$, by $t$-test comparing means. (e) Spectral analysis (power \pm s.e.m.) of the LFP for fourth quarter treated Th-Ga/4/UAS-TrpA1 male flies (black line) and similarly treated controls (data from Th-Gal4/ + and UAS-TrpA1/ + are combined, grey line). Lower and higher gamma $(\gamma)$ ranges are indicated. (f) Data from e binned by $10 \mathrm{~Hz}$ for statistical comparisons. ${ }^{*} P<0.05,{ }^{* *} P<0.01$, by ANOVA. For (c-f), $N=8$ for ThGal4/UAS-TrpA1, $\mathrm{N}=4$ for Th-Gal4/,$+ \mathrm{N}=4$ UAS-TrpA1/ + .

relevant given the convergent evidence from electrophysiological and cellular anatomical studies, indicating the synchronized oscillatory activity of neurons may be abnormal in the brains of patients with schizophrenia (for review see Uhlhaas \& Singer ${ }^{50}$ ). Some researchers have suggested that depressed gamma oscillations may even be a neurophysiological marker for schizophrenia, as neurophysiological responses in this band are selectively depressed in patients with schizophrenia compared with other psychiatric conditions such as personality or mood disorders. ${ }^{51}$ Others have suggested this EEG effect may be a heritable marker (that is, an intermediate phenotype) for schizophrenia, as firstdegree relatives of patients also have reduced gamma-band responses. $^{52}$ The exact relevance of impaired gamma-band activity to this disease is unknown, although recent data indicate it may reflect a deficit of cortical GABA, mainly fast-spiking parvalbumin containing inhibitory interneurons. ${ }^{20}$ Pathology of these interneurons in key cortical regions such as the dorso-lateral PFC represent the most replicated cellular pathology in schizophrenia. ${ }^{53}$ This idea is strengthened by the recent technological advance of being able to selectively target these cortical inhibitory interneurons using optogenetic techniques, providing convincing evidence that fast-spiking GABAergic interneurons drive $25-100-\mathrm{Hz}$ oscillations. ${ }^{54} \mathrm{~A}$ number of psychomimetics (agents that induce psychosis, such as amphetamine) have also very recently been shown to reduce gamma-band activity in the rat cortex. ${ }^{55}$ Of particular note is that upper and lower bands may be pharmacologically dissociable, with serotonergic agonists appearing to reduce both low and high gamma-band activity, while amphetamine appeared to be more selective for gamma power across the upper frequencies. Although gamma in a fly and a mammal need not necessarily reflect similar brain processes, it is tantalizing to find similar frequency attenuations in our model testing the DA ontogeny hypothesis for schizophrenia, as well as in flies acutely exposed to methamphetamine. ${ }^{10}$ In rodent models, tuning of excitatory and inhibitory processes in the PFC is tightly modulated by $D A,{ }^{56}$ which may explain why dopaminergic insults during development compromise gamma-band activity later in life, when DA functions might acquire their eventual adult profile. Our finding of a similar outcome in the fly brain suggests that these effects of DA ontogeny on brain functions may be general, and not necessarily limited to mammalian structures such as the PFC. A broadening of the DA ontogeny hypothesis to include possible effects on oscillatory networks in the brain can now be addressed in Drosophila.

\section{Conflict of interest}

The authors declare no conflict of interest.

Acknowledgements. We thank John McGrath for a critical reading of the manuscript, Angelique Paulk for help with confocal imaging, and James Kesbey for help with HPLC analyses. This work was supported by an Australian Research Council Discovery project grant (DP1093968) and Future Fellowship (FT100100725) to BvS

\section{Author contributions}

BvS and DE planned the study; BC performed the experiments and analyzed the data; BvA conceived and assisted with sleep/arousal experiments; BvS performed the electrophysiology; BvS and DE wrote the paper.

1. Howes OD, Kapur S. The dopamine hypothesis of schizophrenia: version III-the final common pathway. Schizophr Bull 2009; 35: 549-562.

2. van Os J, Kenis G, Rutten BP. The environment and schizophrenia. Nature 2010; 468: 203-212

3. Meyer U, Feldon J. Epidemiology-driven neurodevelopmental animal models of schizophrenia. Prog Neurobiol 2010; 90: 285-326.

4. Cui X, Pelekanos M, Burne TH, McGrath JJ, Eyles DW. Maternal vitamin D deficiency alters the expression of genes involved in dopamine specification in the developing rat mesencephalon. Neurosci Lett 2010; 486: 220-223. 
5. Meyer U, Engler A, Weber L, Schedlowski M, Feldon J. Preliminary evidence for a modulation of fetal dopaminergic development by maternal immune activation during pregnancy. Neuroscience 2008; 154: 701-709.

6. Eyles D, Feldon J, Meyer U. Schizophrenia: do all roads lead to dopamine or is this where they start? Evidence from two epidemiologically informed developmental rodent models. Transl Psychiatry 2012; 2: e81.

7. Burne T, Scott E, van Swinderen B, Hilliard M, Reinhard J, Claudianos C et al. Big ideas for small brains: what can psychiatry learn from worms, flies, bees and fish? Mol Psychiatry 2011; 16: 7-16.

8. Sawamura N, Ando T, Maruyama Y, Fujimuro M, Mochizuki H, Honjo K et al. Nuclear DISC1 regulates CRE-mediated gene transcription and sleep homeostasis in the fruit fly. Mol Psychiatry 2008; 13: 1138-1148.

9. Robbins TW. Arousal systems and attentional processes. Biol Psychol 1997; 45: 57-71.

10. Andretic R, van Swinderen B, Greenspan RJ. Dopaminergic modulation of arousal in Drosophila. Curr Biol 2005; 15: 1165-1175.

11. Kume K, Kume S, Park SK, Hirsh J, Jackson FR. Dopamine is a regulator of arousal in the fruit fly. J Neurosci 2005; 25: 7377-7384.

12. Lebestky T, Chang JS, Dankert H, Zelnik L, Kim YC, Han KA et al. Two different forms of arousal in Drosophila are oppositely regulated by the dopamine D1 receptor ortholog DopR via distinct neural circuits. Neuron 2009; 64: 522-536.

13. Waddell S. Dopamine reveals neural circuit mechanisms of fly memory. Trends Neurosci 2010; 33: 457-464

14. Zhang K, Guo JZ, Peng Y, Xi W, Guo A. Dopamine-mushroom body circuit regulates saliency-based decision-making in Drosophila. Science 2007; 316: 1901-1904.

15. Van Swinderen B, Andretic R. Dopamine in Drosophila: setting arousal thresholds in a miniature brain. Proc Biol Sci 2011; 278: 906-913.

16. Goldman-Rakic PS, Muly EC 3rd, Williams GV. D(1) receptors in prefrontal cells and circuits. Brain Res Brain Res Rev 2000; 31: 295-301.

17. Ye Y, Xi W, Peng Y, Wang Y, Guo A. Long-term but not short-term blockade of dopamine release in Drosophila impairs orientation during flight in a visual attention paradigm. Eur $J$ Neurosci 2004; 20: 1001-1007.

18. Shao L, Shuai Y, Wang J, Feng S, Lu B, Li Z et al. Schizophrenia susceptibility gene dysbindin regulates glutamatergic and dopaminergic functions via distinctive mechanisms in Drosophila. Proc Natl Acad Sci USA 2011; 108: 18831-18836.

19. Brand AH, Perrimon N. Targeted gene expression as a means of altering cell fates and generating dominant phenotypes. Development 1993; 118: 401-415.

20. Gonzalez-Burgos G, Hashimoto T, Lewis DA. Alterations of cortical GABA neurons and network oscillations in schizophrenia. Curr Psychiatry Rep 2010; 12: 335-344.

21. Friggi-Grelin F, Coulom H, Meller M, Gomez D, Hirsh J, Birman S. Targeted gene expression in Drosophila dopaminergic cells using regulatory sequences from tyrosine hydroxylase. J Neurobiol 2003; 54: 618-627.

22. Lundell MJ, Hirsh J. Temporal and spatial development of serotonin and dopamine neurons in the Drosophila CNS. Dev Biol 1994; 165: 385-396.

23. Kitamoto T. Conditional modification of behavior in Drosophila by targeted expression of a temperature-sensitive shibire allele in defined neurons. J Neurobiol 2001; 47: 81-92.

24. Pulver SR, Pashkovski SL, Hornstein NJ, Garrity PA, Griffith LC. Temporal dynamics of neuronal activation by Channelrhodopsin-2 and TRPA1 determine behavioral output in Drosophila larvae. J Neurophysiol 2009; 101: 3075-3088.

25. Evans O, Paulk AC, van Swinderen B. An automated paradigm for Drosophila visual psychophysics. PLoS One 2011; 6: e21619.

26. van Swinderen B, Flores KA. Attention-like processes underlying optomotor performance in a Drosophila choice maze. J Neurobiol 2007; 67: 129-145.

27. Shaw PJ, Cirelli C, Greenspan RJ, Tononi G. Correlates of sleep and waking in Drosophila melanogaster. Science 2000; 287: 1834-1837.

28. Andretic R, Shaw PJ. Essentials of sleep recordings in Drosophila: moving beyond sleep time. Methods Enzymol 2005; 393: 759-772.

29. Chang HY, Grygoruk A, Brooks ES, Ackerson LC, Maidment NT, Bainton RJ et al. Overexpression of the Drosophila vesicular monoamine transporter increases motor activity and courtship but decreases the behavioral response to cocaine. Mol Psychiatry 2006; 11: 99-113.

30. van Swinderen B. Attention-like processes in Drosophila require short-term memory genes. Science 2007; 315: 1590-1593.

31. van Swinderen B, Greenspan RJ. Salience modulates $20-30 \mathrm{~Hz}$ brain activity in Drosophila Nat Neurosci 2003; 6: 579-586.

32. Hillyard SA, Anllo-Vento L. Event-related brain potentials in the study of visual selective attention. Proc Natl Acad Sci USA 1998; 95: 781-787.

33. Mao Z, Davis RL. Eight different types of dopaminergic neurons innervate the Drosophila mushroom body neuropil: anatomical and physiological heterogeneity. Front Neural Circuits 2009; $3: 5$.

34. Gonzalo-Gomez A, Turiegano E, Leon Y, Molina I, Torroja L, Canal I. Ih current is necessary to maintain normal dopamine fluctuations and sleep consolidation in Drosophila. PloS One 2012; 7: e36477.

35. Neckameyer WS, Weinstein JS. Stress affects dopaminergic signaling pathways in Drosophila melanogaster. Stress 2005; 8: 117-131.

36. Neckameyer WS, Quinn WG. Isolation and characterization of the gene for Drosophila tyrosine hydroxylase. Neuron 1989; 2: 1167-1175.

37. Seugnet L, Galvin JE, Suzuki Y, Gottschalk L, Shaw PJ. Persistent short-term memory defects following sleep deprivation in a drosophila model of Parkinson disease. Sleep 2009; 32: 984-992.

38. Broughton SJ, Kitamoto T, Greenspan RJ. Excitatory and inhibitory switches for courtship in the brain of Drosophila melanogaster. Curr Biol 2004; 14: 538-547.

39. Wisor JP, Nishino S, Sora I, Uhl GH, Mignot E, Edgar DM. Dopaminergic role in stimulantinduced wakefulness. J. Neurosci 2001; 21: 1787-1794.

40. Seeman P. Dopamine D2 receptors as treatment targets in schizophrenia. Clin Schizophr Relat Psychoses 2010; 4: 56-73.

41. Vickrey TL, Venton BJ. Drosophila Dopamine2-like receptors function as autoreceptors. ACS Chem Neurosci 2011; 2: 723-729.

42. Seeman P. All roads to schizophrenia lead to dopamine supersensitivity and elevated dopamine D2(high) receptors. CNS Neurosci Ther 2011; 17: 118-132.

43. McGrath JJ, Richards LJ. Why schizophrenia epidemiology needs neurobiology-and vice versa. Schizophr Bull 2009; 35: 577-581.

44. Kapur S. Psychosis as a state of aberrant salience: a framework linking biology, phenomenology, and pharmacology in schizophrenia. Am J Psychiatry 2003 160: 13-23.

45. Fatemi SH, King DP, Reutiman TJ, Folsom TD, Laurence JA, Lee $S$ et al. PDE4B polymorphisms and decreased PDE4B expression are associated with schizophrenia. Schizophr Res 2008; 101: 36-49.

46. van Swinderen B, McCartney A, Kauffman S, Flores K, Agrawal K, Wagner J et al. Shared visual attention and memory systems in the Drosophila brain. PLoS One 2009; 4: e5989.

47. Masseck OA, Hoffmann KP. Comparative neurobiology of the optokinetic reflex. Ann N Y Acad Sci 2009; 1164: 430-439.

48. Rubinstein NJ, Abel LA. Optokinetic nystagmus suppression as an index of the allocation of visual attention. Invest Ophthalmol Vis Sci 2011; 52: 462-467.

49. Tadin D, Kim J, Doop ML, Gibson C, Lappin JS, Blake R et al. Weakened centersurround interactions in visual motion processing in schizophrenia. J Neurosci 2006; 26 : 11403-11412.

50. Uhlhaas PJ, Singer W. Abnormal neural oscillations and synchrony in schizophrenia. Nat Rev Neurosci 2010; 11: 100-113.

51. Lenz D, Fischer S, Schadow J, Bogerts B, Herrmann CS. Altered evoked gamma-band responses as a neurophysiological marker of schizophrenia? Int J Psychophysiol 2011; 79 25-31.

52. Leicht G, Karch S, Karamatskos E, Giegling I, Moller HJ, Hegerl U et al. Alterations of the early auditory evoked gamma-band response in first-degree relatives of patients with schizophrenia: hints to a new intermediate phenotype. J Psychiatr Res 2011; 45 699-705.

53. Lewis DA, Curley AA, Glausier JR, Volk DW. Cortical parvalbumin interneurons and cognitive dysfunction in schizophrenia. Trends Neurosci 2012; 35: 57-67.

54. Sohal VS, Zhang F, Yizhar O, Deisseroth K. Parvalbumin neurons and gamma rhythms enhance cortical circuit performance. Nature 2009; 459: 698-702.

55. Wood J, Kim Y, Moghaddam B. Disruption of prefrontal cortex large scale neuronal activity by different classes of psychotomimetic drugs. J Neurosc 2012; 32: 3022-3031.

56. O'Donnell P. Adolescent onset of cortical disinhibition in schizophrenia: insights from animal models. Schizophr Bull 2011; 37: 484-492.
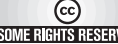

Translational Psychiatry is an open-access journal published by Nature Publishing Group. This work is licensed under the Creative Commons Attribution-NonCommercial-No Derivative Works 3.0 Unported License. To view a copy of this license, visit http://creativecommons.org/licenses/by-nc-nd/3.0/ 